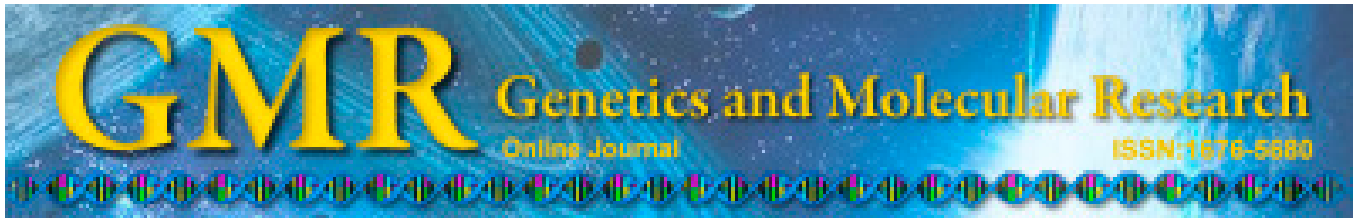

\title{
Association of miR-21 with esophageal cancer prognosis: a meta-analysis
}

\author{
S.-W. Wen ${ }^{1}$, Y.-F. Zhang ${ }^{1}$, Y. Li ${ }^{1}$, Z.-X. Liu ${ }^{2}$, H.-L. Lv' ${ }^{1}$ Z.-H. Li ${ }^{1}$, \\ Y.-Z. Xu' ${ }^{1}$, Y.-G. Zhu ${ }^{1}$ and Z.-Q. Tian ${ }^{1}$ \\ ${ }^{1}$ Department of Thoracic Surgery, \\ Fourth Affiliated Hospital of Hebei Medical University, Shijiazhuang, China \\ ${ }^{2}$ First Hospital of Xinji City, Hebei Province, China \\ Corresponding author: Z.-Q. Tian \\ E-mail: tianziqianng@yeah.net
}

Genet. Mol. Res. 14 (2): 6578-6582 (2015)

Received October 31, 2014

Accepted February 10, 2015

Published June 12, 2015

DOI http://dx.doi.org/10.4238/2015.June.12.12

\begin{abstract}
The present study aimed to explore the relationship between miRNA expression and survival in patients with esophageal cancer (EC) using meta-analysis. We searched PubMed, EMBASE, CNKI, Wanfang, and ISI Web of Science databases without time restrictions, and extracted relevant data, such as the name of first author, publication year, age, gender, number of case, etc. from the studies included. We calculated the pooled hazard ratios (HRs) using the RevMan 5.2 software. A total of five studies involving 504 subjects were included in the meta-analysis, with the purpose of analyzing the association of miRNA-21 expression with EC prognosis. The pooled HR of elevated versus decreased miR-21 expression in EC was 1.87 [95\% confidence interval (CI): 1.37-2.55, P $<0.001]$, with elevated miR-21 expression being associated with poorer prognosis for patients with EC. Our results support a prognostic role for miR21 in EC.
\end{abstract}

Key words: miR-21; Esophageal cancer; Prognosis; Meta-analysis 


\section{INTRODUCTION}

Esophageal cancer (EC) is a multifactor disease associated with varied risk factors (Yano et al., 2014). In humans worldwide, EC is considered the sixth leading cause of cancer deaths (Enzinger and Mayer, 2003). A previous study has shown that there were an estimated 482,300 new cases of EC in 2008 worldwide (Shridhar et al., 2013). The survival rate of EC ranges from 10 (Sant et al., 2003) to 20-30\% (Kirby and Rice, 1994) in different populations.

MicroRNAs (miRNAs) are a class of small noncoding RNAs that are approximately 23 nucleotides in length (Huang et al., 2014). miRNAs are involved in cell development, differentiation, proliferation, and apoptosis through targeting mRNAs (Bao et al., 2014). A previous study (Calin et al., 2004) suggested that over 50\% of miRNA genes are located in fragile sites and cancer associated genomic regions. This result suggested that miRNAs might play a vital role in the pathogenesis of human cancers. Accumulated evidence has indicated that aberrant expression of miRNAs is associated with the prognosis of EC (Hamano et al. 2011; Komatsu et al. 2013). We performed a meta-analysis of the data available from articles published in this field to evaluate the roles of specific microRNAs as prognostic biomarkers in EC.

\section{MATERIAL AND METHODS}

\section{Literature search}

We searched the PubMed, EMBASE, China Knowledge Resource Integrated (CNKI), Wanfang, and ISI Web of Science databases using the search terms "miRNA", or "microRNA" and "esophageal cancer", or "esophageal neoplasm", or "esophageal carcinoma", or "ESCC" and "prognosis" and "survival" without the publication year or language published. In addition, reference lists from the retrieved articles and from review articles were reviewed manually to identify the missing relevant publications.

\section{Eligibility criteria}

Two reviewers independently assessed the eligibility of the retrieved articles. All publications were required to meet the following criteria: 1) reported survival data in patients with EC; 2) provided sufficient data for determining an estimate of hazard ratios (HRs) and $95 \%$ confidence intervals (CIs); and 3) enrolled more than 20 patients. Articles were excluded if relevant data were not available, and studies in which participants did not have documented symptoms or diagnostic evidence of EC were also excluded. To test for between-study heterogeneity, we used the Q- and $\mathrm{I}^{2}$ tests within the RevMan 5.2 software package provided by the Cochrane Collaboration (Oxford, UK; www.cochrane.org).

\section{Data extraction}

Two reviewers independently extracted the data from the included studies. We extracted the following data: name of first author, year of publication, country, number of participants, tumor stage, $\mathrm{HR}$ with $95 \% \mathrm{CI}$, and outcomes. 


\section{Statistical analyses}

Meta-analysis was performed using the RevMan 5.2 software. We used the Q- and $\mathrm{I}^{2}$ tests to examine the heterogeneity between each study. We utilized a fixed effects model to summarize pooled HRs and 95\%CIs using the generic inverse variance method. Publication bias was weighted by a Begg's funnel plot with the Egger's bias indicator test with $\mathrm{P}<0.05$ being considered statistically significant (Song and Gilbody, 1998).

\section{RESULTS}

We identified 256 studies of miRNAs associated with the prognosis of EC from a primary literature search in PubMed, EMBASE, CNKI, Wanfang, and ISI Web of Science databases. In these studies, 7 miRNAs were involved including miR-21, miR-16, miR-31, etc. After screening the titles and abstracts, 236 records were excluded because they involved duplicate publication, review articles, or were irrelevant to the current study. Seven articles were further reviewed in detail. Three of these were further excluded because data were unavailable for further analysis. A total of four articles including five independent studies were eventually included. The clinicopathological characteristics of the eligible studies are summarized in Table 1.

For studies evaluating overall survival in EC, no significant heterogeneity was found $\left(\mathrm{I}^{2}=18 \%, \mathrm{P}=0.30\right)$, therefore, a fixed effects model was applied to calculate the pooled HR (pooled $\mathrm{HR}=1.87,95 \% \mathrm{CI}=1.37-2.55, \mathrm{P}<0.001$; Figure 1).

\begin{tabular}{|c|c|c|c|c|c|c|c|c|}
\hline First author & Publication year & Methods & Population & $\mathrm{N}$ & Stage & $\mathrm{EC}$ & Cutoff & Outcomes \\
\hline Hamano et al. & 2011 & qRT-PCR & Japanese & 98 & I-IV & $\mathrm{U} 48$ & Median & OS \\
\hline Komatsu & 2013 & qRT-PCR & Japanese & 50 & I-IV & U6 & Normal & OS \\
\hline Mathé et al. & 2009 & qRT-PCR & Maryland (USA) & 24 & 0 -IV & U66 & Median & OS \\
\hline Mathé et al. & 2009 & qRT-PCR & Japanese & 33 & 0-IV & U66 & Median & OS \\
\hline Zhao et al. & 2013 & qRT-PCR & Chinese & 178 & I-IV & U6 & Median & OS \\
\hline
\end{tabular}

qRT-PCR = quantitative Real-time Polymerase Chain Reaction; EC $=$ esophageal cancer; OS = overall survival.

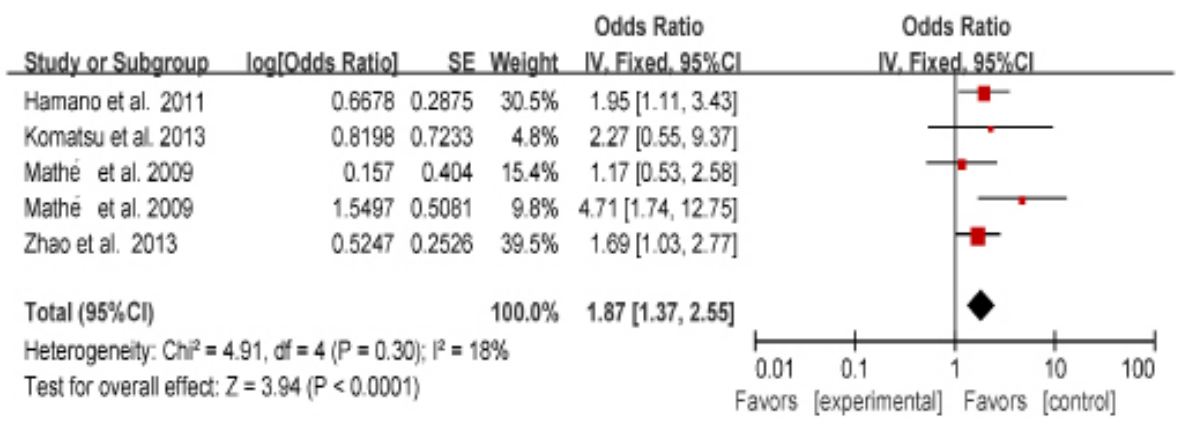

Figure 1. Forest plot of EC and miR-21. The horizontal lines correspond to the study-specific OR and 95\%CI, respectively. The areas of the squares reflect the study-specific weights. The diamond represents the pooled results of $\mathrm{HR}$ and $95 \% \mathrm{CI}$. $\mathrm{EC}=$ esophageal cancer; $\mathrm{OR}=$ odds ratio; $\mathrm{CI}=$ confidence interval; $\mathrm{HR}=$ hazard ratio; $\mathrm{SE}=$ standard error. 
The potential for publication bias within the studies included was evaluated by Begg's plots and the Egger's test. No significant publication biases were found from the results of the Begg's plots (Figure. 2); the Egger's test reached similar conclusions.

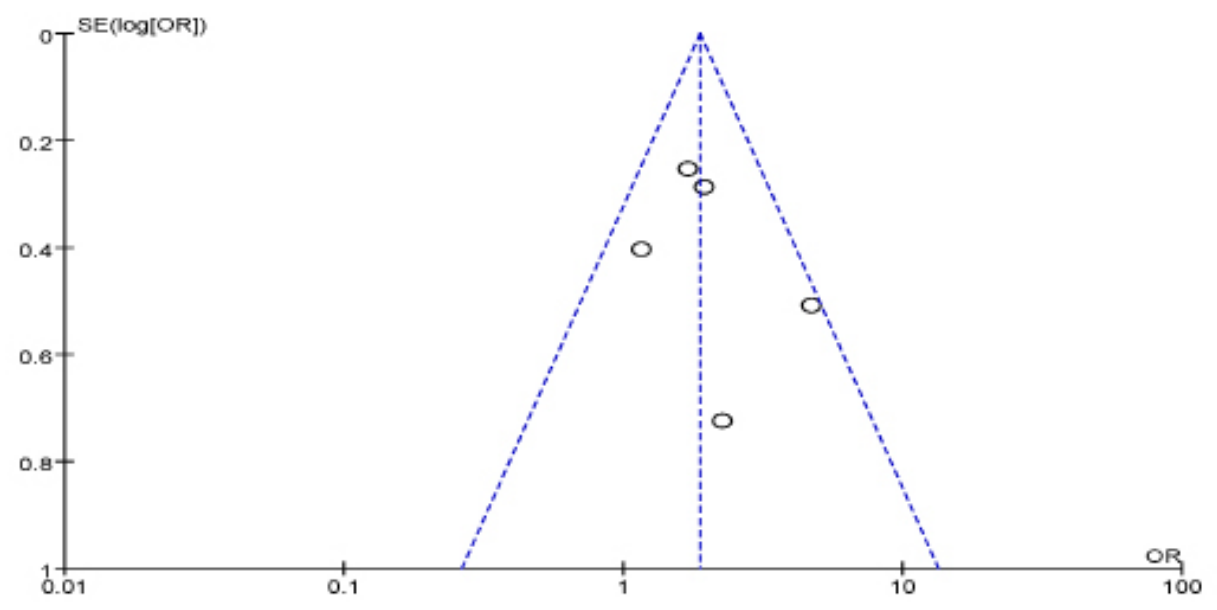

Figure 2. Funnel plot to test for publication bias. Each point represents a separate study for the indicated association. Log OR represents the natural logarithm of the OR. The vertical line represents the mean effects size. OR $=$ odds ratio; $\mathrm{SE}=$ standard error.

\section{DISCUSSION}

In this meta-analysis, we found that miR-21 was associated with EC using the pooled results from five studies published. miR-21 is an oncogenic miRNA that has been shown to be produced at increased levels in EC, and overexpression of miR-21 plays important roles in increasing cell proliferation, migration, invasion, and survival (Li et al., 2013; Wang et al., 2013). Previous studies (Mathé et al. 2009; Komatsu et al., 2013; Wang et al., 2013; Zhao et al., 2013) have suggested that miR-21 downregulation inhibits cell growth and invasion and induces cells to apoptose through targeting of the FASL, TIMP3, and RECK genes. A study by Hamano et al. (2011) also indicated that miR-21 regulates stem cell function, which is involved in the resistance to chemotherapy in EC. In addition, Ma et al. (2011) have also demonstrated that miR-21 was overexpressed in vitro as well as in patients with EC, and promoted cell proliferation by targeting PTEN at posttranscriptional level.

In our study, we found that elevated miR-21 expression predicted poor overall survival in patients with EC (pooled HR =1.87), as was predicted from the above results. However, the conclusions from our study should be tempered because the pooled risks of miR-21 for survival, although statistically significant, were not strong, with global HRs of 1.87.

Several additional limitations of our study must also be mentioned. First, although miR-21 has been widely investigated, only five studies were eligible for pooled analysis, which might affect the results of the present study. Second, whereas ideally a clear threshold should be set for the cutoff value of miRNA levels for specific outcomes in EC, most investigators used the median or mean value in their studies as the cutoff value and the accurate values were different. 
In conclusion, our results support a prognostic role for miR-21 in EC. Further large studies taking into account the effects of gender, ethnicity, and age are warranted to obtain a more robust assessment of the association between miR-21 and the prognosis of EC.

\section{Conflicts of interest}

The authors declare no conflict of interest.

\section{REFERENCES}

Bao B, Azmi AS, Li Y, Ahmad A, et al. (2014). Targeting CSCs in tumor microenvironment: the potential role of ROSassociated miRNAs in tumor aggressiveness. Curr. Stem Cell Res. Ther. 9: 22-35.

Calin GA, Sevignani C, Dumitru CD, Hyslop T, et al. (2004). Human microRNA genes are frequently located at fragile sites and genomic regions involved in cancers. Proc. Natl. Acad. Sci. U. S. A. 101: 2999-3004.

Enzinger PC and Mayer RJ (2003). Esophageal cancer. N. Engl. J. Med. 349: 2241-2252.

Hamano R, Miyata H, Yamasaki M, Kurokawa Y, et al. (2011). Overexpression of miR-200c induces chemoresistance in esophageal cancers mediated through activation of the Akt signaling pathway. Clin. Cancer Res. 17: 3029-3038.

Huang J, Zhang SY, Gao YM, Liu YF, et al. (2014). MicroRNAs as oncogenes or tumour suppressors in oesophageal cancer: potential biomarkers and therapeutic targets. Cell Prolif. 47: 277-286.

Kirby TJ and Rice TW (1994). The epidemiology of esophageal carcinoma. The changing face of a disease. Chest Surg. Clin. N. Am. 4: 217-225.

Komatsu S, Ichikawa D, Tsujiura M, Konishi H, et al. (2013) Prognostic impact of circulating miR-21 in the plasma of patients with gastric carcinoma. Anticancer Res. 33: 271-276.

Li P, Mao WM, Zheng ZG, Dong ZM, et al. (2013). Down-regulation of PTEN expression modulated by dysregulated miR-21 contributes to the progression of esophageal cancer. Dig. Dis. Sci. 58: 3483-3493.

Ma WJ, Lv GD, Tuersun A, Liu Q, et al. (2011). Role of microRNA-21 and effect on PTEN in Kazakh's esophageal squamous cell carcinoma. Mol. Biol. Rep. 38: 3253-3260.

Mathé EA, Nguyen GH, Bowman ED, Zhao Y, et al. (2009). MicroRNA expression in squamous cell carcinoma and adenocarcinoma of the esophagus: associations with survival. Clin. Cancer Res. 15: 6192-6200.

Sant M, Aareleid T, Berrino F, Bielska Lasota M, et al. (2003). EUROCARE-3: survival of cancer patients diagnosed 1990-94 - results and commentary. Ann. Oncol. 14: v61-118.

Shridhar R, Imani-Shikhabadi R, Davis B, Streeter OA, et al. (2013). Curative treatment of esophageal cancer; an evidenced based review. J. Gastrointest. Cancer 44: 375-384.

Song F and Gilbody S (1998). Bias in meta-analysis detected by a simple, graphical test. Increase in studies of publication bias coincided with increasing use of meta-analysis. BMJ 316: 471.

Wang N, Zhang CQ, He JH, Duan XF, et al. (2013). MiR-21 down-regulation suppresses cell growth, invasion and induces cell apoptosis by targeting FASL, TIMP3, and RECK genes in esophageal carcinoma. Dig. Dis. Sci. 58: 1863-1870.

Yano T, Hatogai K, Morimoto H, Yoda Y, et al. (2014). Photodynamic therapy for esophageal cancer. Ann. Transl. Med. 2: 29.

Zhao Y, Schetter AJ, Yang GB, Nguyen G, et al. (2013). microRNA and inflammatory gene expression as prognostic marker for overall survival in esophageal squamous cell carcinoma. Int. J. Cancer 132: 2901-2909. 